\title{
Liquid-Based Cytology Compared to Conventional Cytology for Diagnosis of Cervi- cal Intraepithelial Neoplasia: A Single-Center Experience
}

\author{
Flores-Hernández $\mathrm{L}^{1}$, Ramírez-Uribe $\mathrm{RD}^{2}$, Villegas-González $\mathrm{LF}^{1}$, Serrano-Arévalo $\mathrm{ML}^{1}$, Cordoba- \\ Gonzalez $\mathrm{V}^{3}$ and Barquet-Muñoz SA ${ }^{* 3}$
}

${ }^{1}$ Deparment of Cytology, Instituto Nacional de Cancerología, Tlalpan, Mexico City, Mexico

${ }^{2}$ Deparment of Anatomopathology, Hospital de la Mujer, Secretaria de Salud, Salvador Diaz Miron, Santo Tomas, Mexico City, Mexico

${ }^{3}$ Department of Gynecologic Oncology, Instituto Nacional de Cancerología, Tlalpan, Mexico City, Mexico

${ }^{*}$ Corresponding author: Barquet-Muñoz SA, MD, Department of Gynecologic Oncology, Instituto Nacional de Cancerología. Av. San Fernando 22, Sección XVI, Tlalpan, Mexico City, Mexico, 14080, Fax: 52-55 56-2801-81, Tel: 52-55 56-28-04-00, E-mail: sbarquet@gmail.com

Citation: Flores-Hernández L, Ramírez-Uribe RD, Villegas-González LF, Serrano-Arévalo ML, CordobaGonzalez V, et al. (2018) Liquid-Based Cytology Compared to Conventional Cytology For Diagnosis of Cervical Intraepithelial Neoplasia: A Single-Center Experience. J Gynecol Res 4(2): 206.

doi: 10.15744/2454-3284.4.206

Received Date: June 25, 2018 Accepted Date: September 05, 2018 Published Date: September 07, 2018

\begin{abstract}
Introduction: Cervical carcinoma is the fourth most common gynecologic cancer. Screening assays that include the conventional cytology (CC) have a sensitivity and specificity far from optimal; however, liquid-based cytology (LBC) may overcome some limitations. The objective is to compare the screening accuracy of LBC and CC in individuals suspected of having precancerous lesions.

Materials and Methods: A prospective, single center study, recruited individuals from a tertiary-level center. In all participants, CC and LBC were performed. Clinical, cytologic and histopathologic results were collected. Bethesda System was used and agreement of two of three cytopathologists was necessary to assign a positive or negative result. Cervical biopsy was performed in participants with abnormal findings.

Results: Two-hundred participants were included, CC and LBC were negative for intraepithelial lesion or malignancy, 66 and 56 cases (62.86\% vs $53.33 \%, p=0.162)$ respectively. Cytologic results were confirmed by cervical biopsy, were $13 / 17(76.47 \%)$ were both negative in CC and LBC. Both cytology tests found 7/23 (30.43\%) cases with high-grade squamous intraepithelial lesions. CP and LBC identified $4 / 7$ (57.14\%) carcinomas. Sensitivity and specificity for LBC and CP were (40\% and 76\%) and (51\% and 77\%), respectively. The area under the curve for the LBC was 0.58 (CI95\%, 0.47-0.69) and CC was 0.64 (CI95\%, 0.52-0.75), with no significant difference ( $\mathrm{p}=0.24)$.

Conclusion: Considering no statistically differences in the results and the outlay of LBC, implementation of LBC should be used for molecular-based detection and genotyping when human papillomavirus may offer additional benefits when screening for cervical carcinoma.

Keywords: Liquid-based cytology; Papanicolaou test; Cervical intraepithelial neoplasia; Conventional cytology

List of abbreviations: CC: Conventional Cytology; FDA: Food and Drug Administration; LBC: Liquid-Based Cytology; SD: Standard Deviation; IQR: Interquartile Ranges; AUC: Area under the Curve; LSIL: Low-Grade Squamous Intraepithelial Lesions; HSIL: HighGrade Squamous Intraepithelial Lesions
\end{abstract}

\section{Introduction}

Cervical carcinoma is a widely spread public health problem, representing the fourth most common gynecologic cancer. Notably, $85 \%$ of the global burden occurs in less developed regions of the world, where it is estimated to be $12 \%$ of all cancers diagnosed in women [1]. In Mexico, between the years of 1989 and 2004, the mortality rate for cervical carcinoma was reduced to 2.94 per 100,000 women by the strengthening of screening programs for precancerous cervical lesions and a lower birth rate [2]. Since 2011, cervical cancer is the second cause of cancer-related mortality among Mexican women accounting for $10.4 \%$ of deaths in women with cancer. In contrast, in developed countries the mortality rate associated to cervical cancer has decreased by $70-80 \%$ in the last 
60 years [3]. However, a similar trend has not been observed in developing countries from Latin-American and the Caribbean, due to the scarcity of screening programs, faulty quality control of screening tests, and ineffective health programs for the treatment and follow-up services of the positive screening tests $[4,5]$.

Conventional cytology (CC) for cervical neoplasia screening has an estimated sensitivity ranging from 30 to $87 \%$, specificity of 86 to $100 \%$ and a false negative rate of 25 to $50 \%$, all far from optimal. Thus, the need for research to improve the accuracy of diagnosis [6]. In this line, in 1996 the Food and Drug Administration (FDA) approved an alternative method for sample collection and processing using a liquid-based cytology (LBC) assay with potential for higher sensitivity and specificity rates [7].

Currently most developed countries have substituted CC for LBC as the standard routine test for the detection of precancerous abnormalities, due to the potential use of a single specimen for cytology screening and molecular testing with a higher detection rate of intraepithelial lesions, and lower yield of unsatisfactory samples. However, there is also evidence questioning the proposed superior performance of LBC over the CC. Thus, the aim of the present study is to compare the screening accuracy of liquid-based cytology using the CellPrep Plus method versus the conventional Pap test in patients referred to a tertiary-level medical care center in individuals suspected of having precancerous lesions.

\section{Materials and Methods}

The present prospective, single center study, recruited adult individuals from a community-based, tertiary-level care. A total of 200 participants were included in this study. In all participants, a CC and LBC was performed. Two separate samples were obtained; the first sample acquired was designated for CC and a second sample for the LBC for half of the population, for the second half the sequence was inversed. CC and LBC smears were each assessed by three senior cytopathologist and the Bethesda System was used for description and agreement of at least two of the three cytopathologists was necessary to designate a sample as positive or negative. A cervical biopsy was performed in participants with abnormal findings on gross examination of the cervix. Clinical data, cytologic and histopathologic results were collected from all individuals (including age, number of sexual partners, age at first intercourse, contraceptive methods, smoking status, comorbidities, sample adequacy, transformation zone component, category assigned in accordance to the Bethesda System and final biopsy interpretation report).

Cell samples for cervical cytology, CC and LBC were obtained during speculum examination. For both methods, cells were obtained from the external surface of the cervix and the cervical canal to evaluate the transformation zone. For the conventional Pap test, the Ayre spatula was used to circumferentially scrape the ectocervix followed the endocervix, the sample was smeared onto a slide and fixed with $95 \%$ ethyl alcohol. The commercially available CellPrep Plus LBC (Biodyne, Seongnam, Korea) assay was used for the liquid-based cytology according to the manufacturer's instructions. This study was performed in accordance to the principles of the Declaration of Helsinki and approved by the local ethics committee (Comisión de bioética del Instituto Nacional de Cancerología de México). All participants provided informed consent prior to data collection.

\section{Statistical Analysis}

Normality was assessed using the Kolmogorov-Smirnov test; variables with normal distribution were described using Means \pm standard deviations (SD), whereas for variables without a normal distribution medians and interquartile ranges (IQR) were used. Frequencies and percentages were used to describe categorical data. Correlation between CC and LBC was evaluated using Pearson's or Fisher's exact test, as corresponded. Sensitivity, specificity, negative predictive value, positive predictive value, likelihood ratio, and the area under the ROC curve (AUC) were assessed using cervical biopsy as the reference test. All analyses were two-tailed and p-value $<0.05$ was considered significant. All analyses were performed using STATA 14.1 statistical software.

\section{Results}

A total of 200 participants were included in this study. The main clinical features of participants are summarized in Table 1.

The cytologic analyses for the CC yielded 183 (91.5\%) of satisfactory samples; 122 (61\%) presented endocervical transformation zone; and 122 (61\%) were negative for intraepithelial lesion or malignancy. Epithelial cell abnormality was found in 61 (30.5\%) samples, among these: 14 (7\%) were borderline lesions, 24 (12\%) were low-grade squamous intraepithelial lesions (LSIL) and 10 (5\%) corresponded to high-grade squamous intraepithelial lesions (HSIL). Only 13 (6.5\%) samples were classified as carcinoma. Of note, no significant differences in sample adequacy, detection of transformation zone component, in number of positive and negative samples for intraepithelial lesions as compared to LBC (Table 2).

A comparative study between LBC and CC results was performed, that included 105 individuals with adequate cytological and cervical biopsy samples (Table 3). CC and LBC reports were negative for intraepithelial lesion or malignancy in 66 and 56 cases respectively $(62.86 \%$ vs $53.33 \%, \mathrm{p}=0.162)$. On the other hand, 39 LBC smears and 49 CC, reported for positive intraepithelial lesion or malignancy $(37.14 \%$ vs $46.67 \%$ respectively, $\mathrm{p}=0.162)$.

Cytology results were subsequently confirmed by cervical biopsy, 23 (21.9\%) biopsies resulted in HSIL, from these, 9 (39.13\%) were not detected with CC and $10(43.47 \%)$ with LBC. Conversely, the comparison of the latter results using the reference standard 
yielded a detection rate for carcinoma 4 (54.14\%) and 5 (71.42\%) for CC and LBC, respectively (Table 4).

Sensitivity and specificity for LBC were $40 \%$ and $76 \%$ respectively, while for CC sensitivity $51 \%$ and specificity $77 \%$. The AUC for the LBC was 0.58 (CI 95\%, 0.47-0.69) and for the CC was 0.64 (CI 95\%, 0.52-0.75). No significant difference was noted for AUC between CC and LBC ( $\mathrm{p}=0.24)$ (Table 5).

\begin{tabular}{|c|c|}
\hline & $n=200$ \\
\hline $\operatorname{Age}^{*}$ & $41.3 \pm 13.3$ \\
\hline Biopsy $^{\star *}$ & $121(60.5)$ \\
\hline Age at first intercourse* & $18.3 \pm 3.9$ \\
\hline Number of sexual partners ${ }^{* * *}$ & $2(1-3)$ \\
\hline $\begin{array}{c}\text { Contraception method }^{* *} \\
\text { None } \\
\text { Oral } \\
\text { IUD } \\
\text { Male condom } \\
\text { Female sterilization }\end{array}$ & $\begin{array}{c}66(33) \\
20(10) \\
23(11.5) \\
21(10.5) \\
70(35) \\
\end{array}$ \\
\hline Smoking** & $37(18.5)$ \\
\hline $\begin{array}{c}\text { Comorbidity }^{* *} \\
\text { None } \\
\text { Chronic diseases } \\
\text { Autoimmune diseases }\end{array}$ & $\begin{array}{c}178(89) \\
16(8) \\
6(3)\end{array}$ \\
\hline $\begin{array}{l}{ }^{*} \text { mean (standard deviation) } \\
{ }^{*} \text { absolute frequency (relativ } \\
{ }^{* * *} \text { median (interquartile ran } \\
\text { IUD: Intrauterine device }\end{array}$ & requency) \\
\hline
\end{tabular}

\begin{tabular}{|c|c|c|c|}
\hline & LBC & $\mathrm{CC}$ & $\mathbf{p}$ \\
\hline & n (\%) & n (\%) & \\
\hline $\begin{array}{l}\text { Adequacy } \\
\text { Unsatisfactory } \\
\text { Satisfactory }\end{array}$ & $\begin{array}{c}20(10) \\
180(90)\end{array}$ & $\begin{array}{c}17(8.5) \\
183(91.5)\end{array}$ & 0.605 \\
\hline $\begin{array}{c}\text { Transformation zone component } \\
\text { Absent } \\
\text { Present } \\
\text { Unsatisfactory }\end{array}$ & $\begin{array}{l}70(35) \\
110(55) \\
20(10)\end{array}$ & $\begin{array}{l}61(30.5) \\
122(61) \\
17(8.5)\end{array}$ & 0.477 \\
\hline $\begin{array}{r}\text { Categories } \\
\text { Negative } \\
\text { Abnormality } \\
\text { Unsatisfactory }\end{array}$ & $\begin{array}{c}135(67.5) \\
45(22.5) \\
20(10)\end{array}$ & $\begin{array}{c}122(61) \\
61(30.5) \\
17(8.5)\end{array}$ & 0.191 \\
\hline $\begin{array}{l}\text { Interpretation } \\
\text { Negative } \\
\text { ASC-US, ASC-H, AGC } \\
\text { LSIL } \\
\text { HSIL } \\
\text { Carcinoma } \\
\text { Unsatisfactory }\end{array}$ & $\begin{array}{c}135(67.5) \\
5(2.5) \\
17(8.5) \\
12(6.0) \\
11(5.5) \\
20(10)\end{array}$ & $\begin{array}{c}122(61) \\
14(7) \\
24(12) \\
10(5) \\
13(6.5) \\
17(8.5)\end{array}$ & 0.243 \\
\hline
\end{tabular}

absolute frequency (relative frequency)

ASC-US, ASC-H, AGC: Atypical squamous cells of undetermined significance; high-grade squamous intraepithelial lesion, atypical glandular cells; CC: Conventional cytology; HSIL: Low-grade squamous intraepithelial lesion; LBC: Liquid-based cytology; LSIL: Low-grade squamous intraepithelial lesion Table 2: Comparative results interpretation of Liquid-based cytology and conventional cytology

\begin{tabular}{|c|c|c|c|}
\hline & LBC $^{*}$ & $\mathrm{CC}^{*}$ & p \\
\hline & n (\%) & n (\%) & \\
\hline $\begin{array}{l}\text { Transformation zone component } \\
\text { Absent } \\
\text { Present }\end{array}$ & $\begin{array}{l}34(32.38) \\
71(67.62)\end{array}$ & $\begin{array}{l}30(28.57) \\
75(71.43)\end{array}$ & 0.549 \\
\hline $\begin{array}{l}\text { Categories } \\
\text { Negative } \\
\text { Abnormality }\end{array}$ & $\begin{array}{l}66(62.86) \\
39(37.14)\end{array}$ & $\begin{array}{l}56(53.33) \\
49(46.67)\end{array}$ & 0.162 \\
\hline
\end{tabular}




\begin{tabular}{|c|c|c|c|}
\hline & LBC $^{*}$ & $\mathbf{C C}^{*}$ & $\mathbf{p}$ \\
\hline & $\mathrm{n}(\%)$ & $\mathrm{n}(\%)$ & \\
\hline Interpretation & & & \\
Negative & $66(62.86)$ & $56(53.33)$ & 0.35 \\
ASC-US, ASC-H, AGC & $4(3.81)$ & $10(9.52)$ & \\
LSIL & $13(12.38)$ & $18(17.14)$ & \\
HSIL & $11(10.48)$ & $9(8.57)$ & \\
Carcinoma & $11(10.48)$ & $12(11.43)$ & \\
\hline
\end{tabular}

absolute frequency (relative frequency)

ASC-US, ASC-H, AGC: Atypical squamous cells of undetermined significance; high-grade squamous intraepithelial lesion, atypical glandular cells; CC: Conventional cytology; HSIL: Low-grade squamous intraepithelial lesion; LBC: Liquid-based cytology; LSIL: Low-grade squamous intraepithelial lesion. ${ }^{\star} \mathrm{n}=105$

Table 3: Comparative results interpretation of Liquid-based cytology and conventional cytology; Unsatisfactory samples were excluded

\begin{tabular}{|c|c|c|c|c|c|c|}
\hline \multirow[b]{2}{*}{ Total } & \multicolumn{6}{|c|}{ Histopathologic report } \\
\hline & 17 & 6 & 52 & 23 & 7 & \\
\hline & Negative & Others & LSIL & HSIL & Carcinoma & \\
\hline & n $(\%)$ & n (\%) & n (\%) & n $(\%)$ & n (\%) & \\
\hline Negative & & & & & & Total \\
\hline $\mathrm{CC}^{*}$ & $13(76.47)$ & $5(83.33)$ & $36(69.23)$ & $1(4.35)$ & $1(14.29)$ & 56 \\
\hline $\mathrm{LBC}^{* *}$ & $13(76.47)$ & $6(100)$ & $42(80.77)$ & $4(17.39)$ & $1(14.29)$ & 66 \\
\hline \multicolumn{7}{|c|}{ ASC-US, ASC-H, AGC } \\
\hline $\mathrm{CC}^{*}$ & $1(5.88)$ & $0(0.0)$ & $4(7.69)$ & $4(17.39)$ & $1(14.29)$ & 10 \\
\hline $\mathrm{LBC}^{* *}$ & $1(5.88)$ & $0(0.0)$ & $2(3.85)$ & $0(0.0)$ & $1(14.29)$ & 4 \\
\hline \multicolumn{7}{|l|}{ LSIL } \\
\hline $\mathrm{CC}^{*}$ & $1(5.88)$ & $1(16.67)$ & $11(21.15)$ & $4(17.39)$ & $1(14.29)$ & 18 \\
\hline $\mathrm{LBC}^{* *}$ & $1(5.88)$ & $0(0.0)$ & $6(11.54)$ & $6(26.09)$ & $0(0.0)$ & 13 \\
\hline \multicolumn{7}{|l|}{ HSIL } \\
\hline $\mathrm{CC}^{*}$ & $1(5.88)$ & $0(0.0)$ & $1(1.92)$ & $7(30.43)$ & $0(0.0)$ & 9 \\
\hline $\mathrm{LBC}^{\star *}$ & $2(11.76)$ & $0(0.0)$ & $1(1.92)$ & $7(30.43)$ & $1(14.29)$ & 11 \\
\hline \multicolumn{7}{|l|}{ Carcinoma } \\
\hline $\mathrm{CC}^{*}$ & $1(5.88)$ & $0(0.0)$ & $0(0.0)$ & $7(30.43)$ & $4(57.14)$ & 12 \\
\hline $\mathrm{LBC}^{\star *}$ & $0(0.0)$ & $0(0.0)$ & $1(1.92)$ & $6(26.09)$ & $4(57.14)$ & 11 \\
\hline
\end{tabular}

ASC-US, ASC-H, AGC: Atypical squamous cells of undetermined significance; high-grade squamous intraepithelial lesion, atypical glandular cells; CC: Conventional cytology; HSIL: Low-grade squamous intraepithelial lesion; LBC: Liquid-based cytology; LSIL: Low-grade squamous intraepithelial lesion.

OTHERS: endometrial polyps, vulvar, vaginal and, perineal lesions.

${ }^{\star},{ }^{* *} \mathrm{p}<0.001$

Table 4: Relationship between Liquid-base cytology, Conventional cytology and the biopsy report

\begin{tabular}{|c|c|c|c|c|c|c|c|}
\hline & Sensitivity & Specificity & PPV & NPV & LR+ & LR- & AUC $^{*}$ \\
\hline LBC & 0.40 & 0.76 & 0.89 & 0.20 & 1.69 & 0.19 & 0.58 \\
\hline & & & & & & & $(0.47-0.69)$ \\
\hline CC & 0.51 & 0.77 & 0.92 & 0.25 & 2.17 & 0.65 & 0.64 \\
\hline & & & & & & & $(0.52-0.75)$ \\
\hline
\end{tabular}

AUC: Area Under the Curve; CC: Conventional cytology; LR: Likelihood Ratio; LBC: Liquid-based cytology; NPV: Negative Predictive Value; PPV: Positive Predictive Value

${ }^{*} \mathrm{p}=0.24$

Table 5: Accuracy of Conventional cytology and Liquid-based cytology. Unsatisfactory samples were excluded

\section{Discussion}

Since the introduction of liquid-based cytology there has been divergent consensuses regarding its added benefits. The Australian Health Technology Advisor Committee Report and the Canadian Coordinating Office for Health Technology Assessment argue that LBC increases the detection of cervical lesions and decreases the number of unsatisfactory samples; however, the sensitivity of the test has been controversial, as suggested by the present study findings. The findings of this study, unlike the Australian and Canadian committees, shows no difference in the detection of cervical lesions and the rate of unsatisfactory samples between LBC (sensitivity 51\%) versus the CC (sensitivity 40\%). A Latin American study by Longatto et al. reports superior sensitivity for LBC whereas, Chacho et al. found no significant difference between LBC and the CC for intraepithelial lesions detection $[8,9]$. 
Among the methodological deficiencies found in other studies that may affect the outcome of both types of cytology are: the lesion size, the collection device, the preparation method, the knowledge of the previous histopathological or colposcopy results, intrinsic population characteristics, the follow-up of abnormal tests, related monetary cost $[10,11]$. A differentiating characteristic of the present study is the method of sample harvesting with the shifting of which of the tests received the first sample.

Controversy continues to question the ability of LBC to ease description of specimen adequacy, Longatto et al. and Cheung et al. have reported an improvement in the identification of satisfactory samples Hodgson et al. reported $46 \%$ unsatisfactory samples with double the cost using LBC and found that $31 \%$ of these women will not repeat the test representing a failure in screening [813]. The study results showed no difference detecting the sample adequacy $(\mathrm{p}=0.605)$; among the LBC the principal indicator of unsatisfactory was scant cellularity and, in the CC were obscuring factors (blood and inflammation).

Similar results have been found for HSIL, Taoka et al. reported no difference in their detection using both methods in this study, results are in accordance to their findings [14]. Additionally, Strander et al. found difference between the CC and LBC, but did not statistical significance [15].

\section{Conclusion}

Cervical carcinoma continues to be a burden for developing countries; research continues to lay out new ways to solve the deficit of adequate disease screening. Considering screening performance, the present study concludes non-significant difference in detection rates when using CC and LBC. The greater expense of LBC may prove beneficial when human papillomavirus molecularbased detection and genotyping is advantageous.

\section{References}

1. GLOBOCAN (2012) Cervical cancer. Estimated cancer incidence, mortality and prevalence worldwide in 2012.

2. Lazcano-Ponce E, Palacio-Mejia LS, Allen-Leigh B, Yunes-Diaz E, Alonso P, et al. (2008) Decreasing cervical cancer mortality in Mexico: effect of Papanicolaou coverage, birthrate, and the importance of diagnostic validity of cytology. Cancer Epidemiol Biomarkers Prev 17: $2808-17$.

3. INEGI (2012) Estadisticas De Mortalidad. Cubos Dinamicos Y Conapo 2012. Proyeccciones De La Poblacion De Mexico, $2012-2050$.

4. Ilter E, Midi A, Haliloglu B, Clik A, Yener AN, et al. (2012) Comparison of conventional and liquid-based cytology: do the diagnostic benefits outweigh the financial aspect? Turk J Med Sci 42: 1200-6.

5. Sankaranarayanan R, Gaffikin L, Jacob M, Sellors J, Robles S (2005) A critical assessment of screening methods for cervical neoplasia. Int J Gynaecol Obstet 89: S4-12.

6. Celik C, Gezginc K, Toy H, Findik S, Yilmaz O (2008) A comparison of liquid-based cytology with conventional cytology. Int J Gynaecol Obstet 100: 163-6.

7. Hoda RS, Loukeris K, Abdul-Karim FW (2013) Gynecologic cytology on conventional and liquid-based preparations: a comprehensive review of similarities and differences. Diag Cytopathol 41: 257-78.

8. Longatto Filho A, Pereira SM, Di Loreto C, Utagawa ML, Makabe S, et al. (2005) DCS liquid-based system is more effective than conventional smears to diagnosis of cervical lesions: study in high-risk population with biopsy-based confirmation. Gynecol Oncol 97: 497-500.

9. Chacho MS, Mattie ME, Schwartz PE (2003) Cytohistologic correlation rates between conventional Papanicolaou smears and ThinPrep cervical cytology: a comparison. Cancer 99: 135-40.

10. Bernstein SJ, Sanchez-Ramos L, Ndubisi B (2001) Liquid-based cervical cytologic smear study and conventional Papanicolaou smears: a metaanalysis of prospective studies comparing cytologic diagnosis and sample adequacy. Am J Obstet Gynecol 185: 308-17.

11. Davey E, Barratt A, Irwig L, Chan SF, Macaskill P et al. (2006) Effect of study design and quality on unsatisfactory rates, cytology classifications, and accuracy in liquid-based versus conventional cervical cytology: a systematic review. Lancet 367: 122-32.

12. Cheung AN, Szeto EF, Leung BS, Khoo US, Ng AW (2003) Liquid-based cytology and conventional cervical smears: a comparison study in an Asian screening population. Cancer 99: 331-5.

13. Hodgson W, Kaplan KJ, Rodriguez M, McHale MT, Rose GS, et al. (2005) The impact of converting to liquid-based cervical cytology in a military population. Gynecol Oncol 99: 422-6.

14. Taoka H, Yamamoto Y, Sakurai N, Fukuda M, Asakawa Y et al. (2010) Comparison of conventional and liquid-based cytology, and human papillomavirus testing using SurePath preparation in Japan. Hum cell 23: 126-33.

15. Strander B, Andersson-Ellstrom A, Milsom I, Radberg T, Ryd W (2007) Liquid-based cytology versus conventional Papanicolaou smear in an organized screening program : a prospective randomized study. Cancer 111: 285-91. 


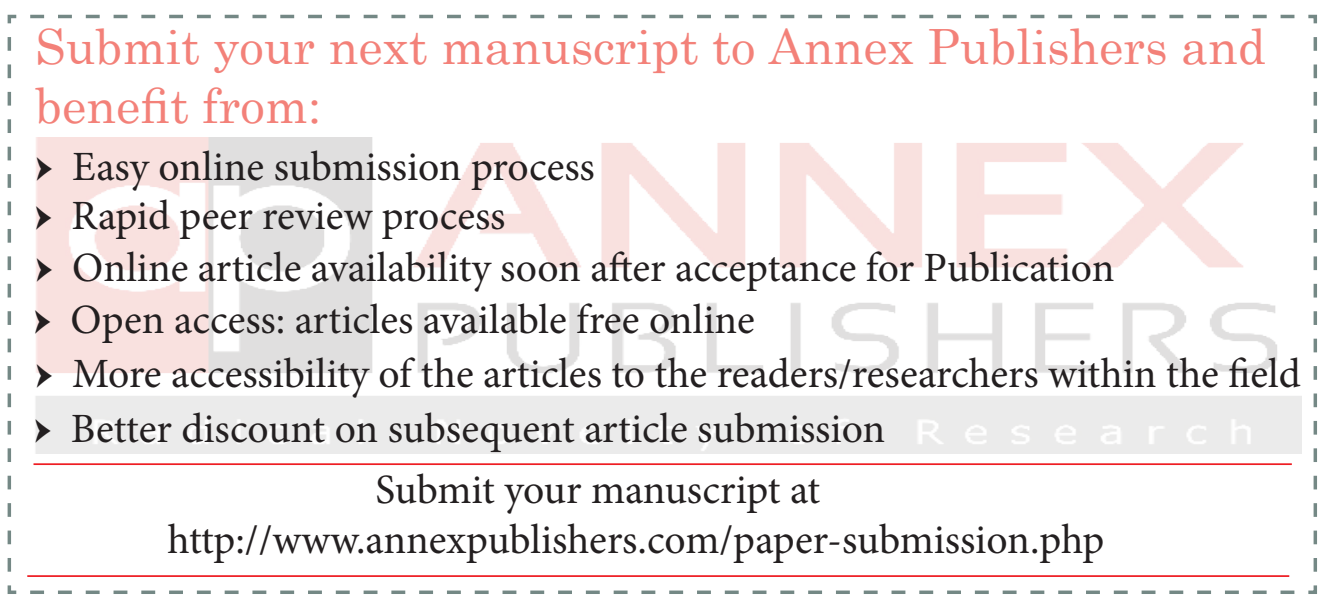

